\title{
Ethnic difference in risk of toxicity in prostate cancer patients treated with dynamic arc radiation therapy
}

\author{
Jose L. Lopez Guerra ${ }^{1,2}$, Raul Matute ${ }^{2,3}$, Fernando Puebla ${ }^{4}$, Alberto Sánchez-Reyes ${ }^{5}$, Beatriz Pontes ${ }^{6}$, Cristina Rubio ${ }^{6}$, \\ Isabel Nepomuceno ${ }^{6}$, Catalina Acevedo ${ }^{2,7}$, Nicolas Isa ${ }^{2,8}$, Rafael Lengua ${ }^{2,9}$, Juan Manuel Praena-Fernandez ${ }^{10}$, \\ Eleonor Rivin del Campo ${ }^{11}$, Maria Jose Ortiz ${ }^{1}$, Ignacio Azinovic ${ }^{2,3}$ \\ ${ }^{1}$ Department of Radiation Oncology, University Hospital Virgen del Rocio, Sevilla - Spain \\ ${ }^{2}$ Master in Advanced Technological Applications in Radiation Oncology, University of Murcia and Grupo IMO Foundation, Madrid - Spain \\ ${ }^{3}$ Department of Radiation Oncology, Oncology Institute of Madrid/Grupo IMO, Madrid - Spain \\ ${ }^{4}$ Department of Radiation Oncology, San Carlos Clinical Hospital, Madrid - Spain \\ ${ }^{5}$ Radiation Physics, Oncology Institute of Madrid/Grupo IMO, Madrid - Spain \\ ${ }^{6}$ Department of Computer Language and Systems, University of Sevilla, Sevilla - Spain \\ ${ }^{7}$ Department of Radiation Oncology, Valle del Lili Foundation, Cali - Colombia \\ ${ }^{8}$ Department of Radiation Oncology, National Cancer Institution, Santiago de Chile - Chile \\ ${ }^{9}$ Department of Radiation Oncology, Hope International Hospital, Guatemala - Guatemala \\ ${ }^{10}$ Methodology Unit, Andalusian Public Foundation for Research Management in Healthcare of Sevilla, Virgen del Rocío University Hospital, \\ Sevilla - Spain \\ ${ }^{11}$ Department of Radiation Oncology, Gustave Roussy Cancer Campus, Villejuif, Paris - France
}

\begin{abstract}
Aims and background: The objective of this study was to assess the influence of ethnicity on toxicity in patients treated with dynamic arc radiation therapy (ART) for prostate cancer (PC).

Methods: From June 2006 to May 2012, 162 cT1-T3 cN0 cM0 PC patients were treated with ART (primary diagnosis, $n=125$; post-prostatectomy/brachytherapy biochemical recurrence, $n=26$; adjuvant post-prostatectomy, $n=11$ ) at 2 institutions. Forty-five patients were Latin Americans and 117 were Europeans. The dose prescribed to the prostate ranged between $68 \mathrm{~Gy}$ and $81 \mathrm{~Gy}$.

Results: The median age was 69 years (range 43-87 years). The median follow-up was 18 months (range 2-74 months). Overall, only 3 patients died, none due to a cancer-related cause. Biochemical recurrence was seen in 7 patients. The rates of acute grade 2 gastrointestinal (GI) and genitourinary (GU) toxicities were $19.7 \%$ and $17 \%$, respectively. Only 1 patient experienced acute grade $3 \mathrm{GI}$ toxicity, whereas 11 patients (6.7\%) experienced acute grade $3 \mathrm{GU}$ toxicity. Multivariate analysis showed that undergoing whole pelvic lymph node irradiation was associated with a higher grade of acute $\mathrm{GI}$ toxicity (OR: 3.46; $\mathrm{p}=0.003)$. In addition, older age was marginally associated with a higher grade of acute GI toxicity (OR: 2.10; $p=0.074$ ). Finally, ethnicity was associated with acute GU toxicity: Europeans had lowergrade toxicity (OR: $0.27 ; p=0.001$ ).

Conclusions: Our findings suggest an ethnic difference in GU toxicity for PC patients treated with ART. In addition, we found that ART is associated with a very low risk of severe toxicity and a low recurrence rate.
\end{abstract}

Keywords: Arc therapy, Data mining, Helical tomotherapy, Prostate cancer, Toxicity

\section{Introduction}

Prostate cancer (PC) is the most frequently diagnosed cancer in men, with 382,000 new cases diagnosed in Europe in 2012; it was the third leading cause of death in men in that

Accepted: February 26, 2015

Published online: May 25, 2015

Corresponding author:

Jose Luis Lopez Guerra, MD, PhD

Department of Radiation Oncology

Virgen del Rocío University Hospital

Manuel Siurot Avenue, s/n. 41013, Sevilla, Spain

chanodetriana@yahoo.es same year (1). The American Cancer Society estimates that 233,000 men are diagnosed in the United States with PC and 29,480 die of it in 2014 (2). Dose escalation in prostate radiotherapy (RT) leads to improved tumor control rates (3), but is limited by the vicinity of surrounding normal tissues, particularly the anorectum and bladder, as several dose-effect relationships for toxicity have been observed. New technologies such as intensity-modulated and image-guided radiation therapy (IGRT) have been shown to decrease acute toxicity in PC $(4,5)$. Dynamic arc IGRT technology accounts for motion to ensure that the target is in the same position for every treatment session $(6,7)$. Innovative radiographic and conebeam computed tomography (CT) modes are integrated with automated repositioning and motion management visualization software to verify that treatments are completely in sync 
with patient breathing, thus increasing treatment accuracy, reducing stress, and increasing patient comfort. The technology provides high-resolution 3-dimensional images to pinpoint tumor sites and adjust positioning when necessary.

The distribution of genotypes depends on the ethnic origin of a population. Differences in the distribution of genotypes between individuals of different ethnicity are an important confounding factor that tends to be undervalued in studies assessing toxicity (8). In the particular case of Europe, although populations inhabiting the Iberian peninsula show substantial genetic homogeneity (9), there are findings which suggest that Northwest African influences exist among the Iberian population and that these differences might increase the risk of false positives in genetic epidemiology studies $(8,10)$.

The purpose of this study was to evaluate the influence of ethnicity on toxicity after definitive arc radiation therapy (ART) for clinically localized and recurrent PC, as well as postprostatectomy adjuvant treatment. In addition, we assessed the clinical results and the prognostic factors for toxicity, with the hypothesis that using IGRT decreases the risk of serious injury to the surrounding normal tissue based on prior studies which show that ART approaches are superior to 3-dimensional conformal radiotherapy (3DCRT) in sparing organs at risk.

\section{Material and methods}

\section{Selection criteria}

The institutional review board approved a multicenter retrospective chart review, which was conducted for individuals with PC treated with ART from June 2006 through May 2012. One hundred and sixty-two cT1-T3 cN0 cMO PC patients were treated with ART (primary diagnosis, $\mathrm{n}=125$; post-prostatectomy/brachytherapy biochemical recurrence, $n=26$ [only 1 patient received brachytherapy as primary treatment]; and adjuvant post-prostatectomy, $n=11$ ) at 2 institutions from different nationalities (Europe and Latin America). Patients were grouped according to the clinical TNM staging system (11) and D'Amico (12) risk classification grouping. Pre-treatment evaluation consisted of documented history and physical examination, including performance status and digital rectal examination. Serum prostate-specific antigen (PSA) values and transrectal ultrasound-guided biopsy of the prostate were obtained before radiation therapy. Abdominal evaluation with $\mathrm{CT} \pm$ magnetic resonance imaging was also required before radiation treatment.

\section{Treatment}

Patients were immobilized in the supine position with the same immobilization device used for 3DCRT. Specific instructions were given regarding daily preparation: a comfortably full bladder (with patients instructed to drink water 30-45 minutes before treatment) and an empty rectum aided by a special diet with instructions from the physician and nurse. Axial images were obtained at 3-mm intervals through the pelvis (from L2 to $10 \mathrm{~cm}$ under the level of the inferior margin of the ischial bone). The outlining of organs at risk was done according to the International Commission on Radiation Units and Measurements, ICRU report 62 (13). The planning target volume (PTV) was defined by a 5- to 10-mm margin around the prostate capsule. The prescription dose covered at least $95 \%$ of the PTV.

All patients were treated with volumetric modulated arc therapy (RapidArc or helical tomotherapy [HT]) and underwent daily megavoltage CT and image-guided verification of the PTV position prior to treatment. The dose prescribed to the prostate ranged between $68 \mathrm{~Gy}$ and $81 \mathrm{~Gy}$, delivered with either hypofractionation (2.5-2.6 Gy/fraction; $n=48$ ) or conventional fractionation (1.8-2 Gy/fraction; $n=114)$. All patients receiving hypofractionation underwent $\mathrm{HT}$. The seminal vesicles received 50-56 Gy, the surgical bed 60-79.2 Gy, and the pelvic lymph nodes 46-50.4 Gy $(n=70)$, when applicable, with conventional fractionation. Neoadjuvant androgen-deprivation therapy (ADT) was given to the majority of intermediate-risk $P C$ patients $(67 \%)$ as well as all high-risk patients who underwent definitive RT. In addition, high-risk patients also received adjuvant ADT. Patients with recurrent PC received ADT at the physician's discretion $(n=8)$.

\section{Patient follow-up}

During the course of RT, patients were seen at least weekly, and more often if needed, for clinical evaluation and disease management. They were evaluated at approximately 1-3 months after completion of therapy and then every 3 months. The follow-up evaluations consisted of a history and physical examination. PSA values were obtained every 3 months for the first year after treatment, every 6 months for 2 years, and annually thereafter. Imaging and additional studies were obtained at the discretion of the treating physician.

\section{Statistical methods}

All data analyses were done using the SPSS statistical software (version 19.0). The primary endpoint was the occurrence of any grade $\geq 2$ acute genitourinary (GU) or gastrointestinal (GI) toxicity within 3 months of RT, scored using the Radiation Therapy Oncology Group (RTOG) scoring system (14). In addition, we evaluated the biochemical failure-free survival defined by the American Society for Therapeutic Radiology and Oncology (ASTRO) and the Phoenix (nadir + 2) definition (15), including any clinical failure defined as local, regional, or distant relapse. Potential risk factors for moderate/severe toxicity (grade $\geq 2$ ) were assessed in univariate logistic regression analysis. Because of the possible confounding effect of clinical factors on toxicity, associations found to be significant in the univariate analysis were adjusted by patient (age, ethnicity), tumor (primary vs. relapse), dosimetry (median dose to organs at risk), and treatment (RT dose, fractionation, whole pelvic lymph node irradiation, RT technique) factors. Multivariate analyses were performed using a logistic regression model, with a stepwise backward elimination procedure. A p value of 0.05 or less was considered statistically significant.

\section{Data mining}

We applied clinical data mining techniques based on association rules (16). The discovery of association rules provides significant and apparently hidden relations among variables 
within a clinical dataset. An association rule can be defined as an implication of the form:

$$
A \Rightarrow B
$$

where $A$ is a set of variables in the clinical dataset and $B$ refers to the level of side effects (moderate/acute).

We applied 2 different families of techniques to extract the rules. On the one hand, we used quantitative techniques where the numerical values of the variables are taken into account. In particular, we applied the C4.5, Ripper and Part algorithms. The C4.5 algorithm is a rule-based classifier and we derive rules from the decision tree that is built (17). The Ripper algorithm is a propositional rule learner (18), and the Part algorithm is a separate-and-conquer rule learner (19). Finally, we used the framework toolkit Weka (20) to execute the algorithms.

On the other hand, we used qualitative techniques where the values of the variables are treated as tags, and numerical variables therefore need to be discretized before the algorithms are applied. We employed Subgroup Discovery (21), a data mining technique which combines predictive and descriptive induction in order to find interesting relationships between variables in a dataset regarding a specific property of interest (level of side effects in our particular case). The task of Subgroup Discovery is therefore to discover subgroups of the population that are statistically "most interesting", i.e., are as large as possible and have the most unusual statistical (distributional) characteristics with respect to the property of interest. We executed this algorithm in the RapidMiner toolkit (22).

\section{Results}

The median age of the patients was 69 years (range 43-87 years; Tab. I). The median follow-up was 18 months (range 2-74 months). The median initial Gleason score was 7 and the median initial PSA was $8.4 \mathrm{ng} / \mathrm{mL}$. There were significant differences in the baseline characteristics between the 2 populations evaluated. Latin-American patients had higher PSA scores, risk classification, radiation doses, longer RT duration, and higher pelvic lymph node irradiation rates than European patients. In contrast, European patients had higher radiation doses per fraction, larger PTVs, larger pelvic volume irradiated, and higher median radiation doses to the bladder and rectum than Latin-American patients (Tab. I). In patients with a primary diagnosis or those receiving adjuvant ART, the median overall survival was 23 months (range, 3.5-92 months). In patients receiving ART for biochemical recurrence, the median survival was 21 months (range, 3-73 months). Overall, only 3 patients died, none of them due to a cancer-related cause. Biochemical recurrence was seen in 7 patients.

\section{Toxicity data}

The rates of acute grade $2 \mathrm{GI}$ and $\mathrm{GU}$ toxicities were $19.7 \%$ and $17 \%$, respectively. Only 1 patient experienced acute grade $3 \mathrm{Gl}$ toxicity, whereas 11 patients (6.7\%) experienced acute grade $3 \mathrm{GU}$ toxicity.

\section{Prognostic factors}

Several factors including dosimetric parameters were evaluated as predictors of acute toxicity in univariate analysis (Tab. II). The radiation dose showed a significant association with grade $\geq 2$ acute $\mathrm{GI}$ and GU toxicity in the univariate analyses ( $p=0.047$ and $p=0.012$, respectively). In addition, whole pelvic lymph node irradiation was also associated with a higher grade of acute GI and GU toxicity $(p=0.003$ and $p=0.015$, respectively). Multivariate analysis (Tab. III) showed that undergoing whole pelvic lymph node irradiation was associated with a higher grade of acute GI toxicity (OR: $3.46 ; p=0.003$ ). In addition, older age was marginally associated with a higher grade of acute GI toxicity (OR: 2.10; $p=0.074$ ). Finally, ethnicity was associated with grade $\geq 2$ acute $\mathrm{Gl}$ and $\mathrm{GU}$ toxicity in univariate analysis ( $p=0.004$ and $p=0.001$, respectively). Latin-American patients showed higher rates of grade $\geq 2 \mathrm{Gl}$ toxicity (36\% vs. $15 \%)$ and GU toxicity ( $41 \%$ and $16 \%)$ than Europeans. However, only the association between ethnicity and a higher grade of acute GU toxicity retained significance in multivariate analysis (OR: 0.27; $\mathrm{p}=0.001$ ).

After data mining analysis, the highest areas under the curve for rules predicting $\mathrm{GI}$ and $\mathrm{GU}$ toxicities ranged from 0.65 to 0.66 and 0.64 to 0.65 , respectively. In terms of $\mathrm{GU}$ toxicity, there were 4 rules which achieved the highest AUC (0.65; Tab. IV). The highest AUC (0.66) for predicting GI toxicity included patients with pelvic lymph node irradiation and PSA $\leq 10 \mathrm{ng} / \mathrm{mL}$ at diagnosis.

\section{Discussion}

This preliminary report shows the feasibility of ART using different radiation techniques for $P C$, including hypofractionation and in the postoperative setting. Our pertinent findings can be summarized as follows. First, we found that ethnicity was associated with acute GU toxicity, with Europeans experiencing lower-grade toxicity. Second, we found that ART was associated with a very low risk of severe toxicity and a low recurrence rate. Interestingly, acute GI and GU toxicities were tolerable without any grade $>3$ side effects. Finally, whole pelvic lymph node irradiation was associated with a higher grade of acute GI toxicity, and older age was marginally associated with a higher grade of acute GI toxicity.

Genetics has been proved to play a role in radiation-induced toxicity (23). Genetic factors partly explain the high interindividual variability in toxicity also when patients have similar characteristics and are treated with the same treatment schedule (24). However, the number of studies assessing ethnicity in PC patients is limited and the overall results are inconclusive $(8,25)$. For example, Lichtensztajn et al $(25)$ reported the outcomes of 90,845 non-Hispanic white, non-Hispanic black, and Asian-American men diagnosed with PC between 2004 and 2010. The population-based cohort of Asian-American men was more likely to have an unfavorable risk profile at diagnosis. In addition, a Spanish multicenter study (8) reported the outcomes of 601 PC patients from 4 geographically distant regions in Spain. The authors observed differences in the distribution of genotypes between individuals of the same ethnicity and concluded that these differences could be an important confounding factor commonly undervalued in typical association studies 
TABLE I - Patient characteristics by ethnicity

\begin{tabular}{|c|c|c|c|c|}
\hline Characteristic & All & Latin Americans ( $n=45$ ) & Europeans $(n=117)$ & $P$ value \\
\hline Median $\left(25^{\text {th }}-75^{\text {th }}\right.$ percentile $)$ & $68.5(63-74.3)$ & $68(59-73.5)$ & $69(63.5-75)$ & 0.299 \\
\hline$<7$ & $65(40)$ & $2(4.4)$ & $63(53.8)$ & \\
\hline 7 & $65(40)$ & $21(46.7)$ & $44(37.6)$ & \\
\hline$>7$ & $32(20)$ & $22(48.9)$ & $10(8.6)$ & $<0.001$ \\
\hline $\mathrm{T} 1$ & $68(41.9)$ & $10(22.2)$ & $58(49.6)$ & \\
\hline $\mathrm{T} 2$ & $65(40.1)$ & $16(35.6)$ & 49 (41.9) & \\
\hline T3 & $27(16.7)$ & $18(40)$ & $9(7.7)$ & \\
\hline $\mathrm{T} 4$ & $2(1.3)$ & $1(2.2)$ & $1(0.8)$ & n.a. \\
\hline \multicolumn{5}{|l|}{$\mathrm{PSA}^{*}(\mathrm{ng} / \mathrm{mL})$} \\
\hline$<10$ & $100(61.7)$ & $27(60)$ & $73(62.4)$ & \\
\hline Low & $34(21)$ & $2(4.4)$ & $32(27.3)$ & \\
\hline Intermediate & $69(42.6)$ & $12(26.7)$ & $57(48.7)$ & \\
\hline High & $59(36.4)$ & 31 (68.9) & $28(24)$ & $<0.001$ \\
\hline \multicolumn{5}{|c|}{ Androgen deprivation therapy $(n=125) * *$} \\
\hline No & $33(26.4)$ & $7(24.1)$ & $26(27.1)$ & \\
\hline Yes & $92(73.6)$ & $22(75.9)$ & 70 (72.9) & 0.753 \\
\hline \multicolumn{5}{|l|}{ Type of radiation } \\
\hline Definitive & $125(77.2)$ & $29(64.4)$ & $96(82)$ & \\
\hline Adjuvant & $11(6.8)$ & $6(13.3)$ & $5(4.3)$ & \\
\hline Salvage & $26(16)$ & $10(22.3)$ & $16(13.7)$ & 0.035 \\
\hline \multicolumn{5}{|l|}{ Fractionation (Gy) } \\
\hline No & $92(56.8)$ & $3(6.7)$ & $89(76.1)$ & \\
\hline Yes & $70(43.2)$ & $42(93.3)$ & $28(23.9)$ & $<0.001$ \\
\hline \multicolumn{5}{|l|}{ Planning target volume $\left(\mathrm{cm}^{3}\right)$} \\
\hline Median $\left(25^{\text {th }}-75^{\text {th }}\right)$ & $131.7(96.9-175.4)$ & $84(59.8-128.7)$ & $140(117.1-190.2)$ & $<0.001$ \\
\hline \multicolumn{5}{|l|}{ Pelvic volume $\left(\mathrm{cm}^{3}\right)(\mathrm{n}=70)$} \\
\hline Median $\left(25^{\text {th }}-75^{\text {th }}\right)$ & $246.2(187.3-708.4)$ & $\begin{array}{c}199(168.9-234.7) \\
(n=42)\end{array}$ & $\begin{array}{c}824.4(643.7-935) \\
(n=28)\end{array}$ & $<0.001$ \\
\hline \multicolumn{5}{|l|}{ Bladder volume $\left(\mathrm{cm}^{3}\right)$} \\
\hline Median $\left(25^{\text {th }}-75^{\text {th }}\right)$ & $141.7(92.7-204.1)$ & $115.8(63.5-177.7)$ & $158.5(104.7-212.7)$ & 0.005 \\
\hline \multicolumn{5}{|l|}{ Rectum volume $\left(\mathrm{cm}^{3}\right)$} \\
\hline Median $\left(25^{\text {th }}-75^{\text {th }}\right)$ & 79.7 (58.6-100.5) & $90.8(71.3-150.7)$ & $77.4(57.5-91.6)$ & 0.001 \\
\hline \multicolumn{5}{|l|}{ Bladder median dose } \\
\hline Median $\left(25^{\text {th }}-75^{\text {th }}\right)$ & $36.5(28-44.8)$ & $32.3(26.7-39.4)$ & $39.7(29.5-47.2)$ & 0.007 \\
\hline \multicolumn{5}{|l|}{ Rectum median dose (Gy) } \\
\hline Median $\left(25^{\text {th }}-75^{\text {th }}\right)$ & $33.1(26.7-38.7)$ & $30.8(27.5-35.1)$ & $34.2(26.6-41.6)$ & 0.022 \\
\hline
\end{tabular}

Categorical variables are represented by absolute frequencies and percentages ( $\mathrm{n}[\%]$ ) and they were analyzed with the chi-square test. Noncategorical variables are represented by the median $\left(25^{\text {th }}-75^{\text {th }}\right.$ percentiles) and were analyzed with the Mann-Whitney $U$ test.

PSA = prostate-specific antigen; PLN, pelvic lymph nodes; PTV = planning target volume; n.a. = not available.

*Values detected at primary diagnosis.

**Includes patients receiving definitive radiation therapy for primary prostate cancer. 
TABLE II - Univariate analysis of factors associated with grade $\geq 2$ acute genitourinary and gastrointestinal toxicity

\begin{tabular}{|c|c|c|c|c|}
\hline Variable & \multicolumn{2}{|c|}{ Acute GU toxicity grade $\geq 2$} & \multicolumn{2}{|c|}{ Acute GI toxicity grade $\geq 2$} \\
\hline$\leq$ median & 1.00 & & 1.00 & \\
\hline$>$ median & 3.45 & 0.345 & 2.01 & 0.082 \\
\hline \multicolumn{5}{|c|}{ PSA at diagnosis (ng/mL) } \\
\hline$>20$ & 0.48 & 0.389 & 0.68 & 0.389 \\
\hline \multicolumn{5}{|l|}{ T stage } \\
\hline $\mathrm{T} 1$ & 1.00 & & 1.00 & \\
\hline $\mathrm{T} 2$ & 1.14 & 0.757 & 1.15 & 0.707 \\
\hline T3 & 0.72 & 0.479 & 0.72 & 0.479 \\
\hline \multicolumn{5}{|l|}{ Gleason score } \\
\hline Low/intermediate & 1.00 & & 1.00 & \\
\hline High & 1.48 & 0.115 & 1.88 & 0.112 \\
\hline \multicolumn{5}{|l|}{ ADT } \\
\hline No & 1.00 & & 1.00 & \\
\hline Yes & 0.49 & 0.294 & 0.69 & 0.294 \\
\hline \multicolumn{5}{|l|}{ Fractionation (Gy) } \\
\hline$\leq 2.00$ & 1.00 & & 1.00 & \\
\hline$>2.00$ & 0.68 & 0.367 & 0.48 & 0.387 \\
\hline \multicolumn{5}{|l|}{ Radiation dose (Gy) } \\
\hline$<$ median & 1.00 & & 1.00 & \\
\hline$\geq$ median & 2.62 & 0.012 & 2.41 & 0.047 \\
\hline \multicolumn{5}{|l|}{ Treatment } \\
\hline Yes & 2.51 & 0.015 & 3.37 & 0.003 \\
\hline \multicolumn{5}{|l|}{ Radiation time (days) } \\
\hline$\leq$ median & 1.00 & & 1.00 & \\
\hline >median & 1.17 & 0.223 & 1.17 & 0.623 \\
\hline \multicolumn{5}{|l|}{ Prostate PTV $\left(\mathrm{cm}^{3}\right)$} \\
\hline$\leq$ median & 1.00 & & 1.00 & \\
\hline$>$ median & 1.21 & 0.225 & 1.41 & 0.295 \\
\hline \multicolumn{5}{|c|}{ Bladder median dose (Gy) } \\
\hline$\leq$ median & NA & & 1.00 & \\
\hline$>$ median & & & 2.41 & 0.011 \\
\hline \multicolumn{5}{|l|}{ Bladder volume (\%) } \\
\hline$\leq$ median & NA & & 1.00 & \\
\hline$>$ median & & & 0.59 & 0.122 \\
\hline Rectum median dose & & & & \\
\hline$\leq$ median & 1.00 & & NA & \\
\hline >median & 2.32 & 0.233 & & \\
\hline Rectum volume (\%) & & & NA & \\
\hline$\leq$ median & 1.00 & & & \\
\hline >median & 0.49 & 0.122 & & \\
\hline
\end{tabular}

$\mathrm{GU}=$ genitourinary; $\mathrm{GI}$ = gastrointestinal; $\mathrm{PSA}=$ prostate-specific antigen; $\mathrm{ADT}$ = androgen deprivation therapy; $\mathrm{OR}=$ odds ratio; $\mathrm{PTV}=$ planning target volume; $\mathrm{PLN}=$ pelvic lymph nodes; $\mathrm{NA}=$ not applicable. 
TABLE III - Multivariate analysis of factors associated with grade $\geq 2$ acute genitourinary and gastrointestinal toxicity

\begin{tabular}{|c|c|c|c|c|c|c|}
\hline Parameter & \multicolumn{3}{|c|}{ Acute GU toxicity grade $\geq 2$} & \multicolumn{3}{|c|}{ Acute GI toxicity grade $\geq 2$} \\
\hline$\leq$ median & & & & 1.00 & & \\
\hline >median & & & - & 3.46 & $1.53-7.84$ & 0.074 \\
\hline Yes & & & - & 2.10 & $0.93-4.74$ & 0.003 \\
\hline \multicolumn{7}{|l|}{ Ethnicity } \\
\hline Latin-American & 1.00 & & & & & \\
\hline European & 0.27 & $0.13-0.59$ & 0.001 & & & - \\
\hline
\end{tabular}

$\mathrm{GU}=$ genitourinary; $\mathrm{Gl}$ = gastrointestinal; $\mathrm{OR}=$ odds ratio; $\mathrm{Cl}$ = confidence interval; $\mathrm{PLN}=$ pelvic lymph nodes .

TABLE IV - Highest areas under the curve of factors associated with grade $\geq 2$ acute genitourinary and gastrointestinal toxicity in multivariate analysis and data mining

\begin{tabular}{|c|c|c|c|}
\hline Analysis & Factors & $\begin{array}{l}\text { Acute GU toxicity grade } \geq 2 \\
\text { AUC }\end{array}$ & $\begin{array}{c}\text { Acute GI toxicity grade } \geq 2 \\
\text { AUC }\end{array}$ \\
\hline \multirow[t]{2}{*}{ Multivariate analysis } & Age (years)* & - & 0.5856 \\
\hline & Ethnicity*** & 0.6452 & - \\
\hline \multirow[t]{5}{*}{ Data mining } & Ethnicity*** \& Gleason score* & 0.6532 & - \\
\hline & Fractionation (Gy) \& Risk $^{+}$ & 0.6532 & - \\
\hline & Radiation time (days) \& Radiation dose* (Gy) & 0.6464 & - \\
\hline & Pelvic lymph node irradiation** \& PSA* (ng/mL) & - & 0.6613 \\
\hline & Pelvic lymph node irradiation** \& Risk $^{+}$ & - & 0.6511 \\
\hline
\end{tabular}

GU = genitourinary; $\mathrm{GI}=$ gastrointestinal; PSA = prostate-specific antigen; $A U C=$ area under the curve.

$* \leq$ median vs. $>$ median.

$* *$ Yes vs. no.

***European vs. Latin-American.

${ }^{\dagger}$ Low \& intermediate vs. high.

${ }^{\ddagger}$ Conventional vs. hypofractionation.

conducted in radiogenomics. Our finding that Europeans experienced lower-grade acute GU toxicity than Latin Americans could be attributable to genetic differences but might also be mediated by sociodemographic, medical, and health behavior factors (26).

HT has been shown to provide a more homogeneous dose distribution, whereas other dynamic arc therapies enable a shorter delivery time (27). IGRT for PC allows adjustment and positional correction of the radiation beams based on daily imaging to account for the variability of the target position. IGRT potentially represents a more accurate form of dose delivery in patients receiving RT for PC. Given the more targeted nature of these treatments, the margins routinely used around the clinical target volume to account for organ motion, and variability of the target position can be further reduced if daily positional correction of the treatment target is performed. Conde-Moreno et al (28) reported the toxicity outcome of PC patients treated with and without IGRT. Those treated with IGRT experienced grade $\geq 2$ proctitis in $4.17 \%$, grade $\geq 2$ rectal bleeding in $2.08 \%$, and grade $\geq 2$ GU toxicity in $8.33 \%$, whereas those treated without IGRT had grade $\geq 2$ proctitis in $19.56 \%$, grade $\geq 2$ rectal bleeding in $15.2 \%$, and grade $\geq 2$ GU toxicity in $15.2 \%$. In addition, lower rates of total and late proctitis, late rectal bleeding, anal fissure, total and acute hematuria, total and acute urinary frequency, and total urinary incontinence were observed in the group treated with IGRT. Our findings showed a very low risk of severe toxicity without any grade $>3$ side effects, even including PC patients treated after surgery or using hypofractionated RT. In this respect, Macias et al (29) had already observed $<1 \%$ 
grade 3 acute complications in PC patients treated with a hypofractionated schedule.

There has been growing interest in the application of data-mining techniques to clinical data in the last 10 years, with a 10-fold increase in the number of papers having the term "data mining" in their title and being referenced in MEDLINE (30). In the present study, we set out to investigate which rules are responsible for moderate/acute RT side effects in order to ascertain whether this approach can refine the prediction of toxicity so that an even more accurate predictive model can be developed. The rules found in this study slightly improved the AUC previously obtained in the logistic regression model. Nevertheless, this is useful information which could open an avenue for future research using this approach to help create new nomograms for predicting not only toxicity but also recurrence and survival outcome.

The study has several limitations, including the relatively small number of patients and the fact that the clinical data were collected and analyzed retrospectively. It will therefore be important to validate our findings in a prospective study with a larger number of patients and a well-defined protocol. We also recognize that differences in baseline characteristics between the 2 populations evaluated, such as radiation doses or disease stage, may have influenced the toxicity outcome. With respect to the data-mining analysis, we acknowledge that our results may only be applicable to this particular series, as external validation is needed to confirm these findings. Therefore, we suggest the use of this data only as a guide. Finally, although it was not the intent of this study, future studies are needed to investigate the mechanism by which ethnicity is associated with the risk of toxicity after RT.

Our findings suggest an ethnic difference in the mechanism behind the association. Therefore, validation of the racial distribution and the association with toxicity in various populations will be an important consideration in toxicity risk evaluation. Aggregating data-mining analyses for prediction of toxicity is beneficial to patient classification and may improve the selection of trial candidates according to the risk of toxicity.

\section{Disclosures}

Financial support: None.

Conflict of interest: The authors have no conflicts of interest related to this study.

\section{References}

1. Ferlay J, Steliarova-Foucher E, Lortet-Tieulent J, et al. Cancer incidence and mortality patterns in Europe: estimates for 40 countries in 2012. Eur J Cancer. 2013;49(6):1374-1403.

2. American Cancer Society. Retrieved September 12, 2014. http://www.cancer.org/cancer/prostatecancer/detailedguide/ prostate-cancer-key-statistics

3. Zelefsky MJ, Pei X, Chou JF, et al. Dose escalation for prostate cancer radiotherapy: predictors of long-term biochemical tumor control and distant metastases-free survival outcomes. Eur Urol. 2011;60(6):1133-1139.

4. Valeriani M, Carnevale A, Osti MF, et al. Hypofractionated intensity-modulated simultaneous integrated boost and imageguided radiotherapy in the treatment of high-risk prostate cancer patients: a preliminary report on acute toxicity. Tumori. 2013;99(4):474-479.

5. Morimoto M, Yoshioka Y, Konishi K, et al. Comparison of acute and subacute genitourinary and gastrointestinal adverse events of radiotherapy for prostate cancer using intensity-modulated radiation therapy, three-dimensional conformal radiation therapy, permanent implant brachytherapy and high-dose-rate brachytherapy. Tumori. 2014;100(3):265-271.

6. Lopez Guerra JL, Isa N, Matute R, et al. Hypofractionated helical tomotherapy using 2.5-2.6 Gy daily fractions for localized prostate cancer. Clin Transl Oncol. 2013;15(4):271-277.

7. Alongi F, Fogliata A, Navarria P, et al. Moderate hypofractionation and simultaneous integrated boost with volumetric modulated arc therapy (RapidArc) for prostate cancer. Report of feasibility and acute toxicity. Strahlenther Onkol. 2012;188(11):990-996.

8. Henríquez-Hernández LA, Valenciano A, Foro-Arnalot $P$, et al. Polymorphisms in DNA-repair genes in a cohort of prostate cancer patients from different areas in Spain: heterogeneity between populations as a confounding factor in association studies. PLoS ONE. 2013;8(7):e69735.

9. Laayouni $\mathrm{H}$, Calafell $\mathrm{F}$, Bertranpetit J. A genome-wide survey does not show the genetic distinctiveness of Basques. Hum Genet. 2010;127(4):455-458.

10. Pino-Yanes $M$, Corrales $A$, Basaldúa $S$, et al. North African influences and potential bias in case-control association studies in the Spanish population. PLoS ONE. 2011;6(3):e18389.

11. American Joint Committee on Cancer. American Joint Committee on Cancer staging manual, $6^{\text {th }}$ ed. New York: Springer; 2002.

12. D'Amico AV, Whittington R, Malkowicz SB, et al. Biochemical outcome after radical prostatectomy, external beam radiation therapy, or interstitial radiation therapy for clinically localized prostate cancer. JAMA. 1998;280(11):969-974.

13. International Commission on Radiation Units and Measurements. ICRU report 62: prescribing, recording, and reporting photon beam therapy. Supplement to ICRU report 50, 2000.

14. Cox JD, Stetz J, Pajak TF. Toxicity criteria of the Radiation Therapy Oncology Group (RTOG) and the European Organization for Research and Treatment of Cancer (EORTC). Int J Radiat Oncol Biol Phys. 1995;31(5):1341-1346.

15. Roach M III, Hanks G, Thames H Jr, et al. Defining biochemical failure following radiotherapy with or without hormonal therapy in men with clinically localized prostate cancer: recommendations of the RTOG-ASTRO Phoenix Consensus Conference. Int J Radiat Oncol Biol Phys. 2006;65(4):965-974.

16. Agrawal R, Imieliński T, Swami A. Mining association rules between sets of items in large databases. SIGMOD Rec. 1993; 22(2):207-216.

17. Salzberg SL. C4. 5: programs for machine learning by J. Ross Quinlan. Morgan Kaufmann Publishers, Inc., 1993. Mach Learn. 1994;16(3):235-240.

18. Cohen WW. Fast effective rule induction. ICML. 1995;95: 115-223.

19. Frank E, Witten IH. Generating accurate rule sets without global optimization. (Working paper 98/2). Hamilton, New Zealand: University of Waikato, Department of Computer Science; 1998.

20. Hall M, Frank E, Holmes G, Pfahringer B, Reutemann P, Witten IH. The WEKA data mining software: an update. ACM SIGKDD Explorations Newsletter. 2009;11(1):10-18.

21. Wrobel S. An algorithm for multi-relational discovery of subgroups. In: Principles of data mining and knowledge discovery. Berlin Heidelberg: Springer; 1997:78-87.

22. Mierswa I, Wurst M, Klinkenberg R, Scholz M, Euler T. Yale: Rapid prototyping for complex data mining tasks. In: Proceedings of the $12^{\text {th }}$ ACM SIGKDD International Conference on Knowledge Discovery and Data Mining. ACM Press; 2006:935-940. 
23. Andreassen $\mathrm{CN}$, Alsner J. Genetic variants and normal tissue toxicity after radiotherapy: a systematic review. Radiother Oncol. 2009;92(3):299-309.

24. Buchholz TA. Finding our sensitive patients. Int J Radiat Oncol Biol Phys. 1999;45(3):547-548.

25. Lichtensztajn DY, Gomez SL, Sieh W, Chung BI, Cheng I, Brooks JD. Prostate cancer risk profiles of Asian-American men: disentangling the effects of immigration status and race/ethnicity. J Urol. 2014;191(4):952-956.

26. Penedo FJ, Dahn JR, Shen BJ, Schneiderman N, Antoni MH. Ethnicity and determinants of quality of life after prostate cancer treatment. Urology. 2006;67(5):1022-1027.

27. Pasquier D, Cavillon F, Lacornerie T, Touzeau C, Tresch E, Lartigau E. A dosimetric comparison of tomotherapy and volumetric modulated arc therapy in the treatment of high-risk prostate cancer with pelvic nodal radiation therapy. Int J Radiat Oncol Biol Phys. 2013;85(2):549-554.

28. Conde-Moreno AJ, Ferrer-Albiach C, Zabaleta-Meri M, JuanSenabre XJ, Santos-Serra A. The contribution of the cone beam Kv CT (CBKvCT) to the reduction in toxicity of prostate cancer treatment with external 3D radiotherapy. Clin Transl Oncol. 2012;14(11):853-863.

29. Macias V, Gonzalez Celador R, Marti-Macia C, Cigarral C, Perez-Romasanta LA. Prognostic factors for acute toxicity in prostate cancer patients treated with high-dose hypofractionated radiotherapy. Clin Transl Oncol. 2013;15(8): 643-651.

30. Han J, Kamber M, Pei J. Data mining: concepts and techniques. Amsterdam Boston Heidelberg: Elsevier/Morgan Kaufmann; 2006. 